\title{
Malignant Brain Tumours in Children : Present and Future Perspectives
}

\author{
James T. Rutka, M.D., Ph.D., FRCSC, FACS, FAANS ${ }^{1-3}$ \\ Division of Neurosurgery, The Hospital for Sick Children, Toronto, Canada \\ The Arthur and Sonia Labatt Brain Tumour Research Centre, ${ }^{2}$ The Hospital for Sick Children, Toronto, Canada \\ Department of Surgery, ${ }^{3}$ The University of Toronto, Toronto, Canada
}

In contrast to many of the malignant tumors that occur in the central nervous system in adults, the management, responses to therapy, and future perspectives of children with malignant lesions of the brain hold considerable promise. Within the past 5 years, remarkable progress has been made with our understanding of the basic biology of the molecular genetics of several pediatric malignant brain tumors including medulloblastoma, ependymoma, atypical teratoid rhabdoid tumour, and high grade glioma/diffuse intrinsic pontine glioma. The recent literature in pediatric neuro-oncology was reviewed, and a summary of the major findings are presented. Meaningful sub-classifications of these tumors have arisen, placing children into discrete categories of disease with requirements for targeted therapy. While the mainstay of therapy these past 30 years has been a combination of central nervous system irradiation and conventional chemotherapy, now with the advent of high resolution genetic mapping, targeted therapies have emerged, and less emphasis is being placed on craniospinal irradiation. In this article, the present and future perspective of pediatric brain malignancy are reviewed in detail. The progress that has been made offers significant hope for the future for patients with these tumours.

Key Words : Medulloblastoma - Ependymoma - Diffuse intrinsic pontine glioma · Atypical teratoid rhabdoid tumour · Molecular genetics · Classification.

\section{INTRODUCTION}

From a historical basis, the survival of children with brain malignancy improved dramatically with the discovery of the role and importance of craniospinal radiation (CSI) in the 1950's by Patterson and Farr which improved survival for children with medulloblastoma from virtually zero to over 50 percent $^{15)}$. For decades afterwards, CSI was the standard of care, and led to a cohort of children with increased survival. However, the advantage of survival was challenged by the deleteri- ous effects of CSI on the developing brain which became increasingly more well recognized. In addition to endocrinopathy, vasculopathy, and possibility of radiation-induced neoplasms, the spectre of profound cognitive impairment was recognized, especially in children receiving CSI under age 5 . This prompted the notion of utilizing reduced dose radiation therapy and supplementation with conventional chemotherapeutics. It took some time, however, before it was fully realized that many chemotherapeutic agents would not cross the blood-brain barrier (BBB); hence, restriction of chemotherapeutics to those which

- Received : February 27, 2018 •Revised : April 26, 2018 •Accepted : April 26, 2018

- Address for reprints : James T. Rutka, M.D., Ph.D., FRCSC, FACS, FAANS

Division of Neurosurgery, The Hospital for Sick Children, 555 University Avenue, Toronto M5G 1X8, Canada

Tel : +1-416-813-6425, Fax : +1-416-813-4975, E-mail : james.rutka@sickkids.ca

This is an Open Access article distributed under the terms of the Creative Commons Attribution Non-Commercial License (http://creativecommons.org/licenses/by-nc/4.0) which permits unrestricted non-commercial use, distribution, and reproduction in any medium, provided the original work is properly cited. 
crossed the BBB became the standard.

From the time of Patterson and Farr when survival for medulloblastoma reached 50 percent, we are now in an era where survivals as high as 80 percent can be expected for average risk patients ${ }^{5}$. Still, however, relapses have been commonplace, and further progress seemingly stalled.

Enter the molecular biology revolution, and our ability now to identify recurrent, non-random genetic alterations which have been highly informative insofar as identifying important genes and molecular pathways that have led to the promise of targeted, novel chemotherapeutics. It is from this vantage point that we find ourselves today. In this regard, major scientific progress has been made in our understanding of the molecular biology and genetics of medulloblastoma, ependymoma, atypical teratoid rhabdoid tumor (ATRT), and diffuse intrinsic pontine glioma (DIPG). Accordingly, this review will focus on the advancements that have been made with respect to these tumour types.

\section{THE FUTURE OF MEDULLOBLASTOMA THERAPY}

A major achievement in our understanding of medulloblastoma biology came with the sub-classification of this aggressive pediatric malignancy into four major subtypes. These subtypes include; wingless (WNT), sonic hedgehog (SHH), group 3 and group 4 medulloblastoma ${ }^{14)}$. This segregation into different subtypes was based on common recurring genetic alterations that were distinct in each subgroup. Much emphasis has been placed recently on further identifying the unique pathway alterations that occur in each subtype. Northcott et al. ${ }^{13)}$ recently described the whole genome landscape of medulloblastoma subtypes from an analysis of 491 patient tumours. They showed that driver mutations could be assigned to the two most malignant forms, group 3 and group 4 medulloblastoma. In particular, hotspot in-frame insertions that target KBTBD4 and activation of PRDN6 were identified ${ }^{13)}$. These and other novel genes identified in this comprehensive, extensive cohort of patients' tumours provide attractive targets for medulloblastoma which have not previously been explored in detail.

Morrissy et al. ${ }^{11)}$ demonstrated substantial special heterogeneity in genetic mutations in medulloblastoma from biopsies taken at different sites within the tumour. This was in stark contrast to the specifically homogeneous transcriptomes that are stable across the tumour, and which enable specific subtyping of medulloblastoma into subgroups. These recent results suggest that both the transcriptome and the genome of medulloblastoma need to be taken into consideration when contemplating successful, targeted therapy for medulloblastoma. This is in distinction to malignant gliomas which demonstrate both genomic and transcriptomic heterogeneity across different regions of a given tumour.

From a molecular genetic analysis of 763 primary tumours, Cavalli et al. ${ }^{4)}$ showed that the four identified subtypes of medulloblastoma could be further subdivided to include 12 different subtypes based on the original four subtypes. The implications of this study are important, as they indicate that groups 3 and 4 medulloblastomas can now be segregated more efficiently with less overlap than before; and that the further subdivisions of $\mathrm{SHH}$ medulloblastomas can be targeted in future clinical trials.

If we are going to treat children with medulloblastoma in clinical trials based on molecular subgrouping in the future, then we need a rapid and reliable method for identifying such subgroups. Recently, Gómez et al. ${ }^{6}$ demonstrated the ability to predict medulloblastoma subgrouping in a rapid and efficient manner using six epigenetic biomarkers. This study examined 913 medulloblastoma samples, and demonstrated a rapid and efficient molecular classification of medulloblastoma using clinically applicable DNA methylation techniques.

Unquestionably, the future of medulloblastoma therapy will be translating the huge repository of molecular data in each subgroup into actionable targeted therapies. As one example, there is an opportunity to "de-escalate" therapy for children with localized WNT medulloblastomas. It is hoped that such a reduction in CSI in this subgroup of patients will be accompanied by improved neurocognitive outcomes and increased quality of life. In addition, efforts should be prioritized for the bad prognosis p53-mutated $\mathrm{SHH}$ and metastatic group 3 medulloblastoma patients. These children have the worst prognosis of all subgroup patients. In this regard, the recent studies mentioned above have already identified putative targets for these patients.

\section{THE FUTURE OF EPENDYMOMA THERAPY}

Ependymoma is another potentially malignant pediatric ma- 
lignancy of the central nervous system in which most variants supratentorial, infratentorial, and spinal - look identical under the microscope. Thankfully, over the past decade, a new understanding has become appreciated of the sub-classification of this tumor type. Now, the supratentorial ependymomas are identified as supratentorial ependymoma-RELA, or supratentorial ependymoma-YAP1; and the posterior fossa group is known as posterior fossa ependymoma-A (PF-A) and posterior fossa ependymoma-B (PF-B $)^{7,10,19)}$. The supratentorial ependymoma RELA subtype is notable for its C11ORF95-RELA gene fusion. The supratentorial ependymoma-YAP1 has fusions affecting this particular oncogene. In general, those children with the YAP1 fusion anomaly have relatively good prognosis when compared to those with the RELA fusion anomaly ${ }^{9)}$.

With respect to the posterior fossa ependymomas, children with PF-A have the worst prognosis as it occurs typically in children less than 3 years of age, is lateral in location, demonstrates histopathological anaplasia, and is usually invasive of the cerebellum and brainstem ${ }^{19)}$. Children with PF-B are older children with midline tumours that rarely invade the cerebellum or brainstem, and are of a less anaplastic histopathological phenotype than are PF-A's. The survival of children with PF-B is considerably better (95\% at 5 years) than children with PF-A (65\% at 5 years).

A recent article by Mack et al. ${ }^{9)}$ demonstrated enhancer regions involving putative oncogene targets and pathways in ependymoma. In their experimental study, the inhibition of these targets led to decreased tumor growth in vitro and in vivo. Much work still needs to be done for ependymoma, as there are no good chemotherapeutics that have been identified for this tumour. In this regard, a recent study has demonstrated that while ependymoma has a fairly stable genome with few, if any, recurrent somatic mutations, its epigenome is quite significantly affected $^{9)}$. This study suggests that drugs that target either DNA or H3K27 methylation could be useful for PF-A's. As histone deacetylase (HDAC) inhibitors are currently being tested within clinical trials for other cancers (for example, vorinostat and panobinostat) the opportunity to use these drugs in posterior fossa ependymoma seems both timely and exciting.

At the present time, and possibly for the foreseeable future, gross total resection of ependymoma is advocated, especially for children with supratentorial and PF-B. Fortunately, for some time, we have been moving away from using CSI to children with ependymoma. It is hoped in the near future, that better pre- clinical models will be developed for ependymoma so that the full extent of potential drug options can be explored in greater detail.

\section{ATRT}

ATRT was first recognized as a distinct CNS tumor type in the late $1980{ }^{\prime 3,12)}$. ATRT is a highly aggressive malignancy of the brain that typically affects young children less than 3 years of age. One of the unique aspects of this malignancy is its ability also to involve other organs, especially the kidneys. The hallmark feature of ATRT is an inactivation of the tumor suppressor gene INI1/SMARCB1 ${ }^{2)}$. After the initial description of ATRTs of the CNS, there were few if any patients with longterm survival. Now, thanks to aggressive multi-modal therapy, some children are surviving ATRT.

Recently, Torchia et al. ${ }^{17)}$ demonstrated the sub-classification of ATRT's through an integrated epigenomic analysis of over 260 ATRTs examining the genomic landscape, through whole genomic and exomic sequencing, gene expression array, $450 \mathrm{~K}$ methylation array, and ultra-high resolution DNA copy number analysis. From this body of work, three subgroups of ATRT have emerged including groups 1,2A, and 2B. Group 1 tumors are predominantly supratentorial in origin, group $2 \mathrm{~A}$ are infratentorial, and group 2B are comprised of a significant number of spinal tumours. In general, although prognosis is not good for any group of ATRT, the group 2 tumors are associated with a worse survival than the group 1 tumors. With the recent molecular characterization of ATRT's, emphasis has been placed on identifying therapeutic targets especially for the group 2 ATRTs. Along these lines, platelet-derived growth factor-beta looks promising as the drug, dasatanib, which targets this molecule, was shown to be effective in reducing the proliferation and growth of a number of group 2-specific ATRT cell lines ${ }^{17,18)}$.

The future of ATRT therapy will undoubtedly relate to our abilities to deploy novel chemotherapeutics towards actionable targets within the molecular biology framework of this highly aggressive tumor. Excitingly, new laboratory and genetically engineered mouse model systems are evolving that will enable us to test this hypothesis. 


\section{PEDIATRIC HIGH GRADE GLIOMA AND DIFFUSE INTRINSIC PONTINE GLIOMAS}

Pediatric high grade gliomas (HGGs) are a heterogeneous group of brain malignancies that typically arise in young children. As a rule, they differ considerably from HGGs in adults from a molecular genetic standpoint. The main molecular alterations within pediatric HGGs include the H3.1 K27M mutation which is found predominantly in DIPG, whereas the H3.3 $\mathrm{K} 27 \mathrm{M}$ is found in midline cerebral locations other than the pons, such as the thalamus ${ }^{8,16}$. The H3.3 G34R/V mutations are found exclusively in the cerebral hemispheres. Several other alterations are found within pediatric HGGs including abnormalities of the BRAF gene through mutations of BRAF600E, and deletion of the CDKN2A. Interestingly, the MET oncogene is often fused. Approximately half of pediatric HGGs demonstrate wild type $B R A F$, normal isocitrate dehydrogenase genes, and no histone methylation alterations making this a highly rich ground for future investigation of these particularly aggressive tumors ${ }^{16)}$.

Without question, the future for DIPG and pediatric HGGs will be a combination of strategies to advance molecular targeting using novel chemotherapeutics, and to enhance delivery of these reagents at higher concentrations across the obstacle of the BBB. In this regard, preclinical progress is being made with convection enhanced delivery strategies ${ }^{20)}$ and the use of magnetic resonance-guided focused ultrasound techniques ${ }^{1)}$.

\section{SUMMARY AND CONCLUSION}

Though there is still much ground to cover with respect to our understanding of pediatric brain malignancies, it is clear that this field has moved rapidly forward. Other tumors not discussed here which are also important to place into future perspectives include malignant germ cell tumors, choroid plexus carcinoma, and tumors previously known as primitive neurectodermal tumors.

Without increasing our understanding of the molecular biology of these particularly aggressive tumors further, we will not make progress. Thankfully, we are making substantial headway with several of the tumours described within this article. It is for this reason that we can remain highly optimistic for the future of treatment of children who have pediatric brain malignancies; and why this particular issue of the Journal of the Ko- rean Neurosurgical Society remains so important and relevant at this time.

\section{CONFLICTS OF INTEREST}

No potential conflict of interest relevant to this article was reported.

\section{INFORMED CONSENT}

Informed consent was obtained from all individual participants included in this study.

\section{References}

1. Alli S, Figueiredo CA, Golbourn B, Sabha N, Yijun M, Bondoc A, et al. : Brainstem blood brain barrier disruption using focused ultrasound. J Control Release, 2018, under revision

2. Biegel JA, Zhou JY, Rorke LB, Stenstrom C, Wainwright $L M$, Fogelgren B : Germ-line and acquired mutations of INI1 in atypical teratoid and rhabdoid tumors. Cancer Res 59 : 74-79, 1999

3. Biggs PU, Garen PD, Powers JM, Garvin AJ : Malignant rhabdoid tumor of the central nervous system. Hum Pathol 18 : 332-327, 1987

4. Cavalli FMG, Remke M, Rampasek L, Peacock J, Shih DJH, Luu B, et al. : Intertumoral heterogeneity within medulloblastoma subgroups. Cancer Cell 31 : 737-754.e6, 2017

5. Gajjar A, Chintagumpala M, Ashley D, Kellie S, Kun LE, Merchant TE, et al. : Risk-adapted craniospinal radiotherapy followed by high-dose chemotherapy and stem-cell rescue in children with newly diagnosed medulloblastoma (St Jude Medulloblastoma-96): long-term results from a prospective, multicentre trial. Lancet Oncol $7: 813-820,2006$

6. Gómez S, Garrido-Garcia A, Garcia-Gerique L, Lemos I, Suñol M, de Torres $C$, et al. : A novel method for rapid molecular subgrouping of medulloblastoma. Clin Cancer Res 24 : 1355-1363, 2018

7. Guerreiro Stucklin AS, Ramaswamy V, Daniels C, Taylor MD : Review of molecular classication and treatment implications of pediatric brain tumors. Curr Opin Pediatr 30 : 3-9, 2018

8. Korshunov A, Ryzhova M, Hovestadt V, Bender S, Sturm D, Capper D, et al. : Integrated analysis of pediatric glioblastoma reveals a subset of biologically favorable tumors with associated molecular prognostic markers. Acta Neuropathol 129 : 669-678, 2015

9. Mack SC, Pajtler KW, Chavez L, Okonechnikov K, Bertrand KC, Wang X, et al. : Therapeutic targeting of ependymoma as informed by oncogenic enhancer profiling. Nature 553 : 101-105, 2018

10. Mack SC, Witt H, Piro RM, Gu L, Zuyderduyn S, Stütz AM, et al. : Epig- 
enomic alterations define lethal CIMP-positive ependymomas of infancy. Nature 506 : 445-450, 2014

11. Morrissy AS, Cavalli FMG, Remke M, Ramaswamy V, Shih DJH, Holgado $\mathrm{BL}$, et al. : Spatial heterogeneity in medulloblastoma. Nat Genet 49 : 780-788, 2017

12. Muller M, Hubbard SL, Provias J, Greenberg M, Becker LE, Rutka JT : Malignant rhabdoid tumour of the pineal region. Can J Neurol Sci 21 : 273-277, 1994

13. Northcott PA, Buchhalter I, Morrissy AS, Hovestadt V, Weischenfeldt J, Ehrenberger T, et al. : The whole-genome landscape of medulloblastoma subtypes. Nature $547:$ 311-317, 2017

14. Northcott PA, Shih DJ, Peacock J, Garzia L, Morrissy AS, Zichner T, et al. : Subgroup specific structural variation across 1,000 medulloblastoma genomes. Nature $488:$ 49-56, 2012

15. Patterson E, Farr RF : Cerebellar medulloblastoma: treated by irradiation of the whole central nervous system. Acta Radiol 39 : 323-336, 1953
16. Sturm D, Bender S, Jones DT, Lichter P, Grill J, Becher O, et al. : Pediatric and adult glioblastoma: multiform (epi)genomic culprits emerge. Nat Rev Cancer 14 : 92-107, 2014

17. Torchia J, Golbourn B, Feng S, Ho KC, Sin-Chan P, Vasiljevic A, et al. : Integrated (epi)-Genomic analyses identify subgroup-specific therapeutic targets in CNS rhabdoid tumors. Cancer Cell 30 : 891-908, 2016

18. Torchia J, Picard D, Lafay-Cousin L, Hawkins CE, Kim SK, Letourneau L, et al. : Molecular subgroups of atypical teratoid rhabdoid tumors in children: an integrated genomic and clinicopathological analysis. Lancet Oncol 16 : 569-582, 2015

19. Witt $H$, Mack SC, Ryzhova M, Bender S, Sill M, Isserlin R, et al. : Delineation of two clinically and molecularly distinct subgroups of posterior fossa ependymoma. Cancer Cell 20 : 143-157, 2011

20. Zhou Z, Singh R, Souweidane MM : Convection-enhanced delivery for diffuse intrinsic pontine glioma treatment. Curr Neuropharmacol 15 : 116-128, 2017 